
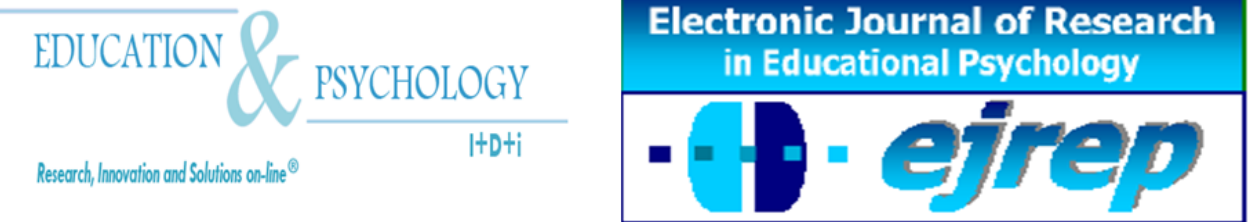

Editorial EOS

\title{
Análisis factorial confirmatorio de las subescalas del $P K B S$-2 para la evaluación de las habilidades sociales y los problemas de conducta en educación infantil
}

\author{
María Fernández, Juan L. Benítez, $M^{\mathrm{a}}$ Carmen Pichardo, \\ Eduardo Fernández, Fernando Justicia, Trinidad García, \\ Ana García-Berbén, Ana Justicia y Guadalupe Alba
}

Dpto. de Psicología Evolutiva y de la Educación

Universidad de Granada

España

Correspondencia: Juan L. Benitez Muñoz. Facultad de Ciencias de la Educación. Campus La Cartuja, s/n, 18071 Granada. España. E-mail: jlbenit@ugr.es

(C) Education \& Psychology $\mathrm{I}+\mathrm{D}+\mathrm{i}$ and Editorial EOS (Spain) 


\section{Resumen}

Introducción. Diferentes investigaciones ponen de manifiesto la importancia de la competencia social como factor de protección frente al comportamiento antisocial. Del mismo modo, alertan de la importancia de contar con instrumentos válidos y fiables que permitan medir estos constructos en edades tempranas.

Método. El objetivo de la investigación es validar las subescalas y proponer un modelo estructural de la Preschool and Kindergarten Behavior Scale - PKBS-2. En el estudio participan 1509 niños y niñas de educación infantil. Los datos se analizan con el programa EQS 6.1 para la realización del análisis factorial confirmatorio y con el SPSS 15.0, para llevar a cabo análisis descriptivos.

Resultados. Los resultados obtenidos señalan buenos índices de ajuste, saturaciones elevadas e índices de consistencia interna adecuados tanto para las subescalas correspondientes a la escala de habilidades sociales como para las correspondientes a la escala de problemas de conducta.

Discusión y conclusiones. Los resultados muestran una elevada fiabilidad en términos de consistencia interna lo que facilita su aplicabilidad en diferentes contextos y específicamente con población española. Se observa que las dos variables que forman el instrumento pueden medirse de manera independiente y por separado lo que permite obtener una información doble con un único cuestionario.

Palabras Clave: educación infantil, medida de problemas de conducta, medida de competencia social, PKBS

Recibido: 10/05/10 Aceptación Inicial: 10/05/10ａceptación Definitiva: 29/07/10 


\title{
Confirmatory factor analysis of the PKBS-2 subscales for assessing social skills and behavioral problems in preschool education
}

\begin{abstract}
Introduction. Different researches point out the importance of social competence as protective factor towards the antisocial behavior. In the same way, alert about the importance of using valid and reliable instruments for measuring such construct in childhood.
\end{abstract}

Method. The goal of this research is to validate the subscales and to propose a structural model of the Preschool and Kindergarten Behavior Scale - PKBS-2. In this study participate 1509 students from preschool education. The data are analyzed with the EQS 6.1 for doing the confirmatory factor analysis and with the SPSS 15.0 for making descriptive analysis.

Results. The results point out good fid indexes, high saturations and adequate internal consistence levels for the subscales of social skills scales as well as for the subscales of behavior problems scales.

Discussion and conclusions. The results show a high reliability in terms of internal consistence. This fact improves the instrument' applicability to different contexts and specifically to Spanish samples. It observed that both variables could be measure independently and separately allowing to obtain double information with just only one questionnaire.

Keywords: preschool education, measure of behaviour problems, measure of social competence, $P K B S-2$

Received: 05/10/10Ｉnitial Acceptance: 05/10/10Ｄefinitive Acceptance: 07/29/10 


\section{Introducción}

El desarrollo socioemocional del niño así como su capacidad para establecer unas relaciones interpersonales positivas con los iguales y con los adultos son temas que, en la actualidad, presentan gran interés tanto en el ámbito social como en el de la investigación científica (Gotzens, Badia, Genovard y Dezcallar, 2010; López de Dicastillo, Iriarte y González-Torres, 2008; Merrell y Harlecher, 2008; Monjas, 1994). La abundante literatura existente en este campo prueba la importancia que se le da hoy a dichos temas. Durante los últimos cincuenta años se constata, además, un cambio en la orientación y contenido de estas publicaciones, pues de haberse enfocado estos temas en la segunda mitad del siglo pasado desde una perspectiva clínica, se ha pasado en la actualidad a estudiarse y trabajarse desde una perspectiva centrada en la educación y formación de la persona así como en la intervención preventiva desde la edad infantil.

Existen razones que justifican el aumento de estudios, teóricos y empíricos, así como el cambio de perspectiva sobre el proceso de socialización de la persona y los problemas de conducta que pueden presentarse. Según Trianes, Sánchez y Muñoz (2001: p. 74), "la competencia social es un aspecto relevante de la construcción personal pues supone aprender a ser y a vivir en un medio socio-cultural” $\mathrm{y}$, al mismo tiempo, "es una estructura psicológica crucial en el funcionamiento psicológico saludable, que presenta complejas relaciones con el desarrollo cognitivo, social y moral puesto que algunos de sus componentes se van adquiriendo en el curso del desarrollo humano". Para López de Dicastillo y colaboradores (2008), la importancia de las relaciones interpersonales en la vida diaria, las características de la sociedad actual que sobrevalora los objetivos y metas individuales por encima de los comunes, los beneficios personales y sociales del desarrollo de comportamientos socialmente competentes así como la necesidad de evitar los problemas originados por la incompetencia social son algunos motivos más que suficientes para originar el interés social por estos temas y para favorecer la actual abundancia de literatura científica sobre los mismos.

No existe una única definición de habilidades sociales aceptada de manera generalizada, aunque la mayor parte de las definiciones incluyen una conducta social positiva y unos resultados y consecuencias igualmente positivos (Merrell, 1995a). Las investigaciones originales en este ámbito han tenido un doble enfoque. Según Furnham 
(1985), en Estados Unidos el entrenamiento en habilidades sociales está enraizado en la psicología clínica y de consulta, pero en Europa esta investigación se debe más a la psicología social. Mientras que la vía norteamericana proviene fundamentalmente del trabajo de terapeutas, como Wolpe, y de los estudios de competencia social de Phillips y Zigler, la investigación europea tuvo su origen en la ergonomía y en la psicología social (Gismero González, 2000). Esta situación inicial ha favorecido la aparición y mantenimiento de una confusión terminológica, empleándose como sinónimas expresiones diferentes. Según López de Dicastillo, Iriarte y González Torres (2008: p. 23), "la competencia social es un concepto multidimensional que incluye el dominio de un conjunto de habilidades sociales conductuales, así como de procesos sociocognitivos (conocimiento social, atribuciones, autoconcepto, expectativas, toma de perspectiva) y de procesos afectivos (expresión, comprensión, regulación de emociones y empatía), dirigidos hacia el logro de una mayor madurez personal y al desarrollo de las cualidades que hacen a las personas y a las sociedades mejores".

Pero el que no haya una definición concreta de competencia social aceptada por toda la comunidad científica, no es obstáculo para que haya unanimidad en el reconocimiento de las características propias de las personas competentes en el ámbito del desarrollo social y de las relaciones interpersonales. Y aunque la mayor parte de los estudios sobre el desarrollo de las habilidades sociales en los niños se han centrado en edades de sujetos escolarizados, existe la convicción de que el aprendizaje de estas habilidades se ha de iniciar desde la etapa de educación infantil por las implicaciones que tiene para el ajuste de la conducta futura (McGinnis y Goldstein, 1990).

La competencia social se enmarca, pues, dentro del comportamiento adaptativo de la persona, mientras que los problemas de conducta se relacionan con comportamientos desadaptativos. Investigaciones realizadas sobre el desarrollo socioemocional en estos últimos años resaltan dos categorías de comportamientos relacionados con los problemas de habilidades sociales: la exteriorización de problemas (conducta agresiva, atención e hiperactividad) y la interiorización de problemas (ansiedad, depresión, timidez) (Cicchetti y Toth, 1991; Merrell, 1995a). Reyna y Brussino (2009) reseñan cómo en un estudio del Ministerio de Salud de la Nación y Unidades Académicas de Psicología de Universidades Nacionales Argentinas del año 
2007 aparece, en una muestra de 5697 niños de 6 a 11 años, que un $14.6 \%$ presenta problemas internos de conducta y un $14.9 \%$ problemas de tipo externo; de lo que deducen la necesidad de dar respuesta a los problemas de comportamiento social infantil desde la prevención y la atención temprana a las conductas desadaptativas.

Un aspecto importante a tener en cuenta, por la importancia de estudiar la relación entre la competencia social y los problemas de conducta en la infancia así como por la necesidad de la detección precoz de dichos problemas, la intervención temprana y el asesoramiento personal, es el problema de la medida de estos constructos a edades tempranas con la consiguiente exigencia de disponer de instrumentos adecuados para dicha medición (Jenetzsch y Merrell, 1996; Merrell, 1995a, 1995b). Existen problemas tales como el relativo vacío o falta de instrumentos para estas edades, muchos de los instrumentos que se utilizan poseen pobres características técnicas y psicométricas, y la necesidad de obtener informaciones objetivas a través de escalas e informantes (Merrell, 1995b). Por todo ello, es necesario abordar los serios problemas referentes a la baja validez y fiabilidad de los instrumentos, así como a la inadecuada estandarización de los procedimientos (Holland y Merrell, 1998; Merrell 2005).

López de Dicastillo, Iriarte y González Torres (2008) ofrecen un amplio listado de instrumentos de evaluación de la competencia social (en inglés), compilado de los trabajos de Caballo (1993), Merrell y Gimpel (1998), Monjas (1994), Paula (2000), Trianes, De la Morena y Muñoz (1999) y Vallés y Vallés (1996), entre otros. Carney y Merrell (2002) hacen una valoración positiva de tres instrumentos diferentes en su artículo referente a la fiabilidad y validez de Preschool and Kindergarten Behavior Scales en su traducción a la lengua castellana: el Child Behavior Checklist/Teacher's Report (CBCL), de Achenbach (1991); el Behavior Assessment System for Children (BASC), de Reynolds y Kamphaus (1992), y el Social Skills Rating System (SSRS), de Gresham y Elliott (1990). Para ellos, cada uno de los tres tiene un poder evaluativo diferente, por lo que necesitarían una valoración especial según la finalidad que se pretenda conseguir con ellos; los dos primeros están más orientados al ámbito de la psicopatología y el tercero a las rutinas de habilidades sociales y problemas de conducta; pero sólo los dos primeros están disponibles en lengua castellana. Merrell y Harlecher (2008) destacan el Child Behavior Checklist-CBCL de Achenbach (1991) como uno de los cuestionarios más utilizados, a pesar de su orientación psicopatológica. 
Análisis factorial confirmatorio de las subescalas del $P K B S-2$ para la evaluación de las habilidades sociales y los problemas de conducta en educación infantil

Y Matson y Wilkins (2009) señalan como los dos instrumentos más usados el Matson Evaluation of Social Skills with Youngsters (MESSI), de Matson, Rotatori y Helsel (1983), y Social Skills Rating System (SSRS), de Gresham y Elliott (1990).

El instrumento Preschool and Kindergarten Behavior Scales (PKBS), de Merrell, ha sido estandarizado con una muestra de 2855 niños de 3 a 6 años (Merrell, 1994a, 1996), y dadas a conocer sus características psicométricas en varias publicaciones, también de otros investigadores. Respecto a la fiabilidad de la prueba, Merrell (1996) obtiene unos índices de .96 para la escala de habilidades sociales y de .97 para la de problemas de conducta. Edwards, Whiteside-Mansell, Conners y Deere (2003), usando una muestra de 1679 niños de 2 a 6 años, obtienen unos índices de .85 para la escala de habilidades sociales y de .97 para la de problemas de habilidades sociales. Y Whiteside-Mansell, Bradley y Mckelvey (2009) obtienen para la información de los padres de niños pequeños unos índices de .90 para la escala de habilidades sociales, de .77 para la interiorización de problemas y de .92 para la exteriorización de problemas; y para la información de los profesores obtienen unos índices de .96 para la escala de habilidades sociales, .92 para la interiorización de problemas y de .97 para la exteriorización de problemas.

La validez del $P K B S$ ha sido demostrada a través de varios procedimientos psicométricos. La validez de contenido, a través de las correlaciones obtenidas, de moderadas a altas, entre los ítems individuales y las puntuaciones totales (Merrell, 1996). La validez de constructo, entre otros sistemas, a través de correlaciones significativas encontradas con otras escalas de valoración del comportamiento (Merrell, 1995b). Y la validez convergente y discriminante se ha mostrado en relación a otras escalas de comportamiento social (Jentzsch y Merrell, 1996), medidas de observación conductual (Winsler y Wallace, 2002) y a escalas de evaluación de hiperactividad (Canivez y Rains, 2002; Canivez y Bordenkircher, 2002).

Carney y Merrell (2002) elaboraron la versión de la escala en español. Las correlaciones ( $\mathrm{r}$ de Pearson) entre las dos versiones de las escalas (inglesa y española) fue de .93 para la escala de Habilidades sociales y .94 para la escala de Problemas de 
conducta. La consistencia interna (alfa de Cronbach) fue semejante: .93 para la escala de Habilidades sociales y .96 para la escala de Problemas de conducta.

Para dar respuesta a la necesidad de contar con instrumentos adaptados para evaluar la competencia social y los problemas de conducta de la población española infantil, el objetivo de la presente investigación ha sido analizar las características psicométricas de la traducción y adaptación al español de la versión revisada de la Preschool and Kindergarten Behavior Scale-PKBS (Merrell, 2002).

\section{Método}

\section{Participantes}

La muestra está formada por 1509 alumnos (741 niños y 768 niñas) entre los 3 y los 5 años de edad (media $=3.78 ; \mathrm{dt}=.815)$ pertenecientes a 27 centros de educación infantil de Granada capital y su área metropolitana. La misma muestra se utiliza para realizar la validación cruzada de los resultados repartiéndose de forma aleatoria el total de sujetos entre la muestra de derivación y la muestra de confirmación.

Tabla 1. Distribución de la muestra según sexo y edad

\begin{tabular}{llcccc} 
& & 3 años & 4 años & 5 años & Total \\
\cline { 2 - 6 } Niños & $\mathrm{N}$ & 370 & 152 & 219 & 741 \\
& $\%$ del total & $24,5 \%$ & $10,1 \%$ & $14,5 \%$ & $49,1 \%$ \\
Niñas & $\mathrm{N}$ & 403 & 153 & 212 & 768 \\
& $\%$ del total & $26,7 \%$ & $10,1 \%$ & $14,0 \%$ & $50,9 \%$ \\
Total & $\mathrm{N}$ & 773 & 305 & 431 & 1509 \\
& $\%$ del total & $51,2 \%$ & $20,2 \%$ & $28,6 \%$ & $100,0 \%$ \\
\hline
\end{tabular}

\section{Instrumento}

La escala utilizada es la traducción y adaptación al español de la versión revisada de la Preschool and Kindergarten Behavior Scale - PKBS (Merrell, 2002). La escala tiene por objetivo desarrollar un instrumento psicométricamente válido para la evaluación de las habilidades sociales y los problemas de comportamiento en niños 
entre los 3 y los 6 años de edad (ver anexo 1). Está formada por 76 ítems que permiten evaluar dichos parámetros repartidos en dos escalas diferenciadas.

Por un lado, la escala de habilidades sociales (34 ítems) comprende a su vez tres subescalas:

1. Cooperación Social (12 ítems). Refleja un ajuste social en las relaciones con los adultos y los iguales. Los ítems valoran características conductuales que son importantes para el seguimiento de instrucciones, cooperación y compromiso con los iguales y un adecuado autocontrol.

2. Interacción Social (11 ítems). La subescala refleja conductas $\mathrm{y}$ características que son importantes para la adquisición y mantenimiento de la aceptación y la amistad de los otros. Los ítems incluyen adaptación social en la relación con los iguales y los adultos.

3. Independencia social (11 ítems). Refleja los comportamientos y las características que son importantes en la realización de independencia social dentro del ámbito del grupo de iguales. Se incluye la evaluación de algunas habilidades sociales relacionadas con adecuada separación de los adultos cuidadores, confianza y asertividad en la interacción con otros.

Por otra parte, la escala de problemas de conducta (42 ítems) evalúa problemas de comportamiento que han demostrado ser comunes en la infancia temprana/preescolar. Estos problemas de conducta se evalúan a través de 2 subescalas:

1. Exteriorización de Problemas (27 ítems). Los ítems describen comportamientos perturbadores activos y no controlados por parte de los niños, reflejados hacia los demás.

2. Interiorización de Problemas (15 ítems). Los ítems de la subescala evalúan problemas emocionales y conductuales relacionados con ansiedad, temor o hipersensibilidad emocional.

Los comportamientos de los niños, evaluados por el profesorado o el cuidador, se valoran según una escala de tipo Likert de 4 puntos que oscila entre 0 (nunca) y 3 (con frecuencia). De esta forma, puntuaciones altas en la escala de habilidades sociales reflejan un mejor desarrollo de las mismas, en tanto que, puntuaciones bajas en la escala de problemas de conducta reflejan la ausencia de conductas desadaptadas. 


\section{Procedimiento}

El primer paso de la presente investigación consistió en la traducción y adaptación de la escala original en inglés a español. Tras la revisión lingüística y la maquetación se procedió a realizar un estudio piloto para detectar posibles errores o dificultades de comprensión de los ítems traducidos así como para valorar el comportamiento de la escala.

Tras el estudio piloto, y sin detectar errores o dificultades, se procedió a contactar con diversos centros para conocer el interés en la administración del cuestionario. Tras el contacto inicial, y en reuniones con el profesorado, se les informó sobre los objetivos del estudio, las características de la escala y sobre el procedimiento para contestar al cuestionario. Una vez el profesorado dio su consentimiento para participar en el estudio se informó a los padres. La información a los padres se llevó a cabo mediante una reunión informativa y mediante cartas para darles a conocer el objetivo y naturaleza del estudio para, finalmente, pedirles el consentimiento para que los hijos participasen en el estudio.

Los cuestionarios fueron repartidos al profesorado en el mes de noviembre, tras pasar un período de dos meses desde la reunión inicial. Tal período se lleva a cabo para lograr el máximo nivel de adaptación entre profesorado y alumnado, y para que el docente tenga el suficiente conocimiento del alumnado que le permita contestar con mayor facilidad a los ítems presentes en el cuestionario. Tras un mes los investigadores recogieron los cuestionarios.

\section{Análisis Estadístico}

Los datos se analizaron con el programa Statistical Package for the Social Sciences (SPSS) versión 15.0, utilizando técnicas descriptivas para el análisis de la curtosis y asimetría asociadas a los ítems del cuestionario. Por otro lado, y para la realización del análisis confirmatorio se utilizó el programa EQS 6.1 (Bentler, 2004). El análisis factorial confirmatorio realizado parte del modelo a priori establecido por el autor y tomando como base análisis realizados por otros investigadores (Carney y Merrell, 2002, 2005; Edwards, Whiteside-Mansell, Conners, y Deere, 2003; Reyna y 
Brussino, 2009). El análisis factorial confirmatorio se realiza con el objetivo de validar y confirmar la bondad de ajuste de las escalas del $P K B S$-2. Para la evaluación del ajuste se utiliza la prueba del ji-cuadrado que ayuda a indicar el conveniente ajuste datosmodelo aunque, debido a su sensibilidad al tamaño muestral (Eren, 2009; Tomás y Oliver, 2004), fue necesario complementarla con otros índices. Índices de ajuste absoluto que ayudan a determinar el grado en el que el modelo predice la matriz de covarianzas observada: Goodness of Fit Index (GFI $\geq .90)$, Root Mean Square Residual (RMSR $\leq .08)$ y Root MSE of Approximation $(\mathrm{RMSEA} \leq .08)$. Índices de ajuste incremental que permiten comparar el ajuste de modelo en relación con el modelo de referencia que no estipula ninguna relación entre variables: Normed Fit Index (NFI $\geq .90)$, Comparative Fit Index (CFI $\geq .90)$ y Adjusted Goodness of Fit Index (AGFI $\geq .90)$. E índices de parsimonia que señalan la simplicidad del modelo en relación con el número de parámetros libres: Akaike Information Criterion (AIC) que informa de una mayor parsimonia cuanto más se aproxima a cero (Lévy y Varela, 2006).

\section{Resultados}

El análisis descriptivo de los ítems de las escalas de Habilidad Social y de Problemas de Conducta muestra una distribución adecuada de los ítems de cada escala. Así, se observa que los ítems de la escala de habilidades sociales presentan asimetría negativa en tanto que los de la escala de problemas de comportamiento presentan asimetría positiva, tal y como cabría esperar. El valor de los resultados obtenidos apoya la utilización del estimador de máxima verosimilitud en los análisis confirmatorios posteriores (Edwards et al., 2003; Muthen y Kaplan, 1985).

Para valorar la factibilidad del estudio factorial se analizan los valores de la matriz de correlaciones de ambas escalas en relación con la prueba de esfericidad de Bartlett y el índice de Kaiser-Meyer-Olkin (KMO). Los valores de la prueba de esfericidad de Bartlett para la escala de habilidades sociales $\left(\chi^{2}=32046.66, p<.001\right)$ y para la escala de problemas de conducta $\left(\chi^{2}=47088.60, p<.001\right)$ señalan que la matriz de correlaciones de la población no es una matriz identidad. Del mismo modo, los valores 
de la prueba KMO para ambas escalas (.965 y .975, respectivamente) señalan que la factorización de la matriz de correlaciones es factible.

\section{Análisis Factorial Confirmatorio de las subescalas de Habilidades Sociales}

\section{Subescala de Cooperación Social}

Los resultados del modelo a priori de la subescala muestran índices de ajuste no adecuados $(A G F I=.877$ y $R M S E A=.094)$. La solución estandarizada permite observar la saturación factorial del ítem y el error asociado a la varianza no explicada del mismo. Como procedimiento de depuración se opta por eliminar aquellos ítems con una saturación $<.40$ y error $>.80$. Del mismo modo, y para conocer la relación entre variables que ayuden a ajustar el modelo, se utiliza el Test de Lagrange que permite evaluar los parámetros que fijados a cero provocarían una mejora significativa del ajuste global. Según los criterios anteriores no se elimina ningún ítem y se establece la covariación de los residuales del ítem 30 “acepta turnos para utilizar los juguetes $u$ otros objetos" y del ítem 25 "comparte sus juguetes y otras pertenencias", y la covariación de los residuales del ítem 2 "es cooperativo" con los correspondientes a los ítems 12 "utiliza bien su tiempo libre" y 25. Los resultados obtenidos tras las modificaciones realizadas señalan índices de ajuste adecuados (Tabla 2): $\chi^{2}=434.815$, $g l=51, \quad p<.001 ; \quad A I C=332.815 ; \quad N F I=.959 ; \quad C F I=.964 ; \quad G F I=.952 ; \quad A G F I=.927 ;$ $R M S R=.031 ; R M S E A=.071 ; \alpha=.925$.

\section{Subescala de Interacción Social}

En relación con el modelo inicial establecido para la subescala de interacción social éste presenta índices de ajuste inadecuados $(N F I=.876 ; C F I=.881 ; G F I=.873$; RMSEA =.123). Tras considerar los criterios anteriormente citados no se elimina ningún ítem. Por otro lado, se establece la relación entre los residuales del ítem 21 "invita a otros niños a jugar" con los correspondientes a los ítems 33 "es sensible a los problemas de los niños" y 34 "muestra afecto por otros niños" respectivamente, y a la covariación entre el ítem 15 "pide ayuda a un adulto cuando la necesita" con los residuales de los ítems 14 "participa en las conversaciones familiares o escolares" y 24 "busca el apoyo de un adulto cuando se encuentra mal". Los resultados obtenidos tras 
considerar los cambios producen unos índices de ajuste adecuado (Tabla 2): $\chi^{2}=425.260, \quad g l=40, \quad p<.001 ; \quad A I C=345.260 ; \quad N F I=.950 ; \quad C F I=.954 ; \quad G F I=.947 ;$ $A G F I=.912 ; R M S R=.043 ;$ RMSEA $=.079 ; \alpha=.907$.

\section{Subescala de Independencia Social}

La subescala de independencia social tampoco presenta un ajuste adecuado en relación con el modelo a priori $(N F I=.878 ; C F I=.883 ; G F I=.895 ; R M S E A=.111)$. Siguiendo los criterios establecidos se procede a la eliminación del ítem 1 "es independiente cuando juega y trabaja" e ítem 26 "defiende sus derechos". Además, se establecen las covariaciones entre los residuales: del ítem 11 "otros niños le invitan a jugar" con los del ítem 6 "es aceptado y cae bien a los niños", del ítem 3 "ríe y se divierte con otros niños" con el ítem 4 "juega con distintos niños", y del ítem 18 "se adapta bien a diferentes circunstancias" con los ítems 8 "intenta hacer la tarea sin pedir ayuda" y 31 "se muestra seguro en situaciones sociales". Los resultados obtenidos tras considerar los cambios producen unos índices de ajuste adecuado (Tabla 2): $\chi^{2}=194.321, \quad g l=23, \quad p<.001 ; \quad A I C=148.321 ; \quad N F I=.969 ; \quad C F I=.973 ; \quad G F I=.972 ;$ $A G F I=.945 ;$ RMSR $=.039 ;$ RMSEA $=.070 ; \alpha=.878$.

Tabla 2. Subescalas de Habilidades Sociales, índices de ajuste y fiabilidad

\begin{tabular}{ccccccccccc}
\hline Subescala & $\chi^{2}$ & $g l$ & $A I C$ & NFI & CFI & GFI & AGFI & RMSR & RMSEA & $\alpha$ \\
\hline Cooperación Social & $434.815^{*}$ & 51 & 332.815 & .959 & .964 & .952 & .927 & .031 & .071 & .925 \\
Interacción Social & $425.260^{*}$ & 40 & 345.260 & .950 & .954 & .947 & .912 & .043 & .079 & .907 \\
Independencia Social & $194.321^{*}$ & 23 & 148.321 & .969 & .973 & .972 & .945 & .039 & .070 & .878 \\
\hline$* p<.001$ & & & & & & & & & &
\end{tabular}




\title{
Análisis Factorial Confirmatorio de las subescalas de Problemas de Conducta
}

\author{
Subescala de Exteriorización de Problemas
}

Los resultados del modelo a priori de la subescala muestran índices de ajuste no adecuados $(N F I=.837 ; C F I=.846 ; G F I=.739 ; R M S E A=.100)$. De acuerdo con los criterios asumidos previamente no se procede a la eliminación de ninguno de los ítems de la subescala. No obstante, se establece la relación entre los residuales del: ítem 1 "actúa impulsivamente $y$ sin pensar" con los correspondientes a los ítems 16 "desobedece las normas", 20 "es muy activo, incapaz de quedarse quieto" y 25 "es inquieto y travieso"; los residuales del ítem 26 "insulta a los compañeros" con los correspondientes a los ítems 3 "se burla y se ríe de los demás" y 21 "es vengativo con los demás niños"; los residuales del ítem 29 "maltrata o intimida a otros compañeros" con los correspondientes a los ítems 26 y 21; los residuales del ítem 37 “lloriquea y se queja" con los de los ítems 13 "grita o chilla cuando se enfada" y 35 "es muy caprichoso"; y por último, los residuales del ítem 7 "tiene un temperamento fuerte" con los residuales de los ítems 13 y 19 "se deben hacer las cosas a su manera". Los resultados obtenidos tras considerar los cambios producen unos índices de ajuste adecuado (Tabla 3): $\chi^{2}=2989.890, g l=306, \quad p<.001 ; \quad A I C=2377.890 ; \quad N F I=.906$; $C F I=.915 ; G F I=.902 ; A G F I=.877 ; R M S R=.043 ;$ RMSEA $=.076 ; \alpha=.967$.

\section{Subescala de Interiorización de Problemas}

La subescala de interiorización de problemas tampoco presenta un ajuste adecuado en relación con el modelo a priori $(N F I=.789 ; C F I=.796 ; G F I=.813$; $R M S E A=.123)$. Siguiendo los criterios asumidos no se encuentran ítems que los cumplan. Sin embargo, se establecen las relaciones entre: los residuales del ítem 12 "evita jugar con otros niños" y los de los ítems 4 " no responde al afecto de los otros", 17 "tiene dificultades para establecer amistades" y 28 "se aísla de los demás compañeros"; los residuales del ítem 5 "está demasiado apegado al padre o al profesor" y de los ítems 18 "es miedoso y asustadizo" y 24 "se resiste a ir al colegio"; los residuales del ítem 2 "finge estar enfermo cuando tiene miedo" y del ítem 23 "se queja de molestias o enfermedades"; y por último, los residuales del ítem 28 y 17. Los resultados obtenidos tras considerar los cambios producen unos índices de ajuste 
Análisis factorial confirmatorio de las subescalas del $P K B S-2$ para la evaluación de las habilidades sociales y los problemas de conducta en educación infantil

adecuado (Tabla 3): $\chi^{2}=823.403, g l=82, p<.001 ; A I C=659.403 ; N F I=.919 ; C F I=.927$;

$G F I=.934 ; A G F I=.903 ; R M S R=.048 ; R M S E A=.077 ; \alpha=.905$.

Tabla 3. Subescalas de Problemas de Conducta, indices de ajuste y fiabilidad

\begin{tabular}{ccccccccccc}
\hline Subescala & $\chi^{2}$ & $g l$ & $A I C$ & NFI & CFI & GFI & AGFI & RMSR & RMSEA & $\alpha$ \\
\hline Exteriorización Problemas & $2989.890^{*}$ & 306 & 2377.890 & .906 & .915 & .902 & .877 & .043 & .076 & .967 \\
Interiorización Problemas & $823.403 *$ & 82 & 659.403 & .919 & .927 & .934 & .903 & .048 & .077 & .905 \\
\hline$* p<.001$ & & & & & & & & & &
\end{tabular}

\section{Discusión y Conclusiones}

Merrell (1995a) manifiesta que el hecho de utilizar modelos de ecuaciones estructurales conduce a conclusiones más fuertes sobre la dirección de la causalidad entre algunas escalas y las subescalas, o entre las escalas entre ellas mismas. En este estudio se ha pretendido conocer esas relaciones en profundidad, a la vez que comprobar la validez y fiabilidad de la Preschool Kindergarten Behavior Scale, de Merrell (2002), por ello se utiliza además del SPSS, el EQS.

Tras realizar el análisis factorial confirmatorio del cuestionario se puede concluir que se obtienen resultados que indican una adecuada bondad de ajuste. Las subescalas en las que se centra el cuestionario presentan unos índices de ajuste adecuados tras realizar los análisis, a la vez que un Alpha de Cronbach elevado, muy cercano al 1. La Independencia Social es la que tiene el $\alpha$ más bajo y, pese a todo, es de .878. Las demás sobrepasan el .900, siendo algunos valores de .967, como ocurre con la variable Exteriorización de Problemas. Sucede lo mismo en diferentes estudios realizados por diversos investigadores. Por ejemplo, Carney y Merrell (2002) obtienen un índice de fiabilidad de .93 en la escala de Habilidades Sociales y un índice de .94 en Problemas de Conducta.

Los datos anteriores nos indican que la escala es un instrumento adecuado para medir dichas variables, ofreciendo una alta validez y fiabilidad. 
Si se tiene en cuenta el modelo original, son dos los ítems que una vez realizados los análisis se ha procedido a eliminar. Éstos son, dentro de la subescala de Independencia Social, el 1 (es independiente cuando juega y trabaja), y el 26 (defiende sus derechos). Comparando el modelo original con los 1509 niños de la muestra española, en un primer momento no ajusta de manera adecuada ninguna variable, lo que cambia cuando se realizan los análisis factoriales confirmatorios. Se constata la importancia de adaptar los diversos instrumentos a las características de la población de los diferentes países, sobre todo si se traducen a otro idioma. Si esto no se lleva a cabo, no se tendrá certeza de que los datos obtenidos describan correctamente la población diana.

Un aspecto muy positivo que ofrece el cuestionario, y que se observa tras obtener los diferentes índices de ajuste, es que las dos variables que lo forman, pueden medirse de manera independiente y por separado, es decir, tienen entidad propia de manera individual, es como si tuviéramos dos cuestionarios en uno, lo que completa en gran medida la información que de él obtenemos (Merrell, 1995a). Merrel (1995a), tras realizar un estudio sobre la validez del PKBS obtiene una importante relación negativa entre la escala Habilidades Sociales y la subescala Interiorización de Problemas. Los niños que obtienen puntuaciones elevadas en Interiorización de Problemas presentan normalmente déficits de habilidades sociales, pudiendo constatarse tras evaluaciones realizadas por dicho instrumento. Además, los índices de correlación entre la escala de Habilidades Sociales y la de Problemas de Conducta que obtienen Carney y Merrell (2002) son significativos.

Otros estudios que confirman los datos obtenidos son el de Jentzsch y Merrell (1996) o el de Merrell (1995b). En ambos casos se comprueba una alta correlación entre las diferentes escalas a la vez que una elevada fiabilidad y validez, los diferentes ítems que forman las subescalas miden el mismo constructo y se constata una elevada consistencia interna. Los análisis que realizan se centran en la validez externa comparando el PKBS con instrumentos como el MESSY (Mattson Evaluation of Social Skills with Youngsters de Matson, Rotari, y Helsel, 1983), el SSBS (School Social Behavior Scales, de Merrell, 1993) o el SSCSA (Scale of Social Competence and School Adjustment de Walker y McConnell, 1995). 
Un aspecto que hace que los datos obtenidos en este estudio adquieran una mayor validez, es el hecho de que los cuestionarios fueron cumplimentados en 27 centros de la provincia de Granada, lo cual quiere decir que los evaluadores fueron diferentes y las características de los centros muy dispares. Este hecho le da un mayor peso a los resultados, ya que no son datos de una muestra en concreto sino que abarcan un muestreo aleatorio, reforzando la objetividad y superando limitaciones existentes en otros estudios (Reyna y Brussino, 2009). A pesar de este aspecto positivo, es conveniente observar qué ocurre cuando se comparan los datos con los de los padres de los niños, sin ser evaluados éstos solamente por los maestros, utilizando así diferentes fuentes de evaluación (Jentzsch y Merrell, 1996 y Merrell, 1995b).

Debemos ser conscientes de la importancia que tiene el análisis de fiabilidad y validez de los cuestionarios que utilizamos para estudiar el comportamiento humano. Cada estudio llevado a cabo no es sino un paso más para asegurar que los datos que obtenemos de ellos nos pueden aportar cierta confianza, pero es cierto que es necesario llevar a cabo muchas más investigaciones para poder confirmar al 100\% los datos aquí obtenidos. Sólo en este caso se llevará a cabo una adecuada prevención de los problemas de conducta, siendo éste nuestro objetivo último.

\section{Agradecimientos}

La investigación presentada en el artículo está financiada por el Proyecto $\mathrm{I}+\mathrm{D}+\mathrm{i}$, Estudio longitudinal de los efectos del programa aprender a convivir en el desarrollo de la competencia social y la prevención del comportamiento antisocial (EDU200911950) financiado por el Ministerio de Ciencia e Innovación y los Fondos Feder (Fondo Social Europeo). 


\section{Referencias}

Achenbach T.M. (1991). Child Behavior Checklist. Burlington, V.T.: Center for Children, Youth, and Families.

Bentler, P. M. (2004). EQS 6 Structural Equation Program Manual. Multivariate Software, Encino: CA.

Canivez, G.L. y Bordenkircher, S.E. (2002). Convergent and divergent validity of the adjustement scales for children and adolescent and preschool and kindergarten behavior scales. Journal of Psychoeducational Assessment, 20, 30-45.

Canivez, G.L. \& Rains, J.D. (2002). Construct validity of the Adjustement Scales for Children and Adolescents and the Preschool and Kindergarten Behavior Scales: Convergent and divergent evidence. Psychology in the Schools, 39, 621-633.

Carney, A. G. y Merrell, K. W. (2002). Reliability and comparability of a Spanishlanguage form of the Preschool and Kindergarten Behavior Scales. Psychology in the Schools, 39, 367-373.

Carney, A. G. y Merrell, K. W. (2005). Teacher ratings of Young children with and without ADHD: Construct validity of two child behavior rating scales. Assessment for Effective Intervention, 30 (3), 65-75.

Cicchetti, D. y Toth, S.L. (1991). A developmental perspective on internalizing and externalizing disorders. En D. Cicchetti y S.L. Toth (Eds.). Internalizing and externalizing expressions of dysfunction (pp. 1-19). Hillsdale, NJ: Erlbaum.

Edwards, M.C., Whiteside-Mansell, L., Conners N.A. y Deere, D. (2002). The utility of the Preschool and Kindergarten Behavior Scales in assessing child behavior in an at-risk sample. Journal of Psychoeducational Assessment, 21 (1), 16-31.

Eren, A. (2009). Exploring the effects of changes in future time perspective and perceived instrumentality on graded performance. Electronic Journal of Research in Educational Psychology, 19, 7(3), 1217-1248.

Furnham, A. (1985). Social skills training: A european perspective. En L. L'Abate y M.A. Milan (eds.). Handbook of Social Skills Training and Research. New York: John Willey and sons, 555-580.

Gismero González, E. (2000). Manual de la Escala de Habilidades Sociales (EHS). Madrid: TEA Ediciones, S.A.

Gotzens, C., Badia, Ma., Genovard, C. y Dezcallar, T. (2010). Estudio comparativo de la gravedad atribuida a las conductas disruptiva en el aula. Electronic Journal of Research in Educational Psychology, 20, 8(1), 33-58. 
Gresham, F.M. y Elliott, S.N. (1990). Social Skills Rating System Manual. Circle Pines: MN American Guidance Services.

Holland, M.L. y Merrell, K.W. (1998). Social-emotional characteristics of preschoolage children referred for Child-Find screening and assessment. A comparative study. Research in Developmental Disabilities, 19, 167-179.

Jentzsch, C. E., y Merrell, K. W. (1996). An investigation of the construct validity of the Preschool and Kindergarten Behavior Scales. Diagnostique, 21(2), 1-15.

Lévy, J. P. y Varela, J. (2006). Modelización con estructuras de covarianzas en ciencias sociales. España: Netbiblo.

López de Dicastillo, N., Iriarte, C. y González Torres, M.C. (2008). Competencia social y educación cívica. Concepto, evaluación y programas de intervención. Madrid: Editorial Síntesis, S.A.

Matson, J. L., Rotari, A. F. y Helsel, W.J. (1983). Development of a rating scale to measure social skills in children: The Matson Evaluation of Social Skills with Youngsters (MESSY). Behaviour Research and Therapy, 21, 335-340.

Matson, J.L. y Wilkins, J. (2009). Psychometric testing methods for children's social skills. Reasearch in Developmental Disabilities, 30, 249-274.

Merrell, K.W. (1993). School Social Behavior Scales. Brandon, VT: Clinical Psychology Publishing.

Merrell, K.W. (1994a). Preschool and Kindergarten Behavior Scales. Austin, TX: PROED.

Merrell, K.W. (1994b). Assessment of behavioral, social, and emotional problems: Direct and objective methods for use with children and adolescents. White Plains, NY: Longman.

Merrell, K. W. (1995a). An investigation of the relationship between social skills and internalizing problems in early childhood: Construct validity of the Preschool and Kindergarten Behavior Scales. Journal of Psychoeducational Assessment $13,230-240$.

Merrell, K. W. (1995b). Relationships Among Early Childhood Behavior Rating Scales: Convergent and Discriminant Construct Validity of the Preschool and Kindergarten Behavior Scales. Early Education y Development, 6 (3), 253-264. 
Merrell, K.W y Gimpel, G.A. (1998). Social skills of children and adolescents. Conceptualization, assessment, treatment. New Jersey: Lawrence Erlbaum Associates.

Merrell, K. W. (2002). Preschool and Kindergarten Behavior Scales. Austin (Texas): Pro-ed.

Merrell, K. W. (2003). Preschool and Kindergarten Behavior Scales, second edition: Spanish language version. Austin, TX: PRO-ED.

Merrell, K.W. y Harlecher, J.E. (2008). Behavior rating scales. En R.P. Archer y S.R. Smith (Eds.). Personality Assessment. New York: Routledge/Taylor y Francis.

Monjas, I. (1994). Evaluación de la competencia social y las habilidades sociales en la edad escolar. En M.A. Verdugo (Dir.). Evaluación curricular. Una guía para la intervención psicopedagógica. Madrid: Siglo XXI.

Muthen, B. B., y Kaplan, D. (1985). A comparison of some methodologies for the factor analysis of non-normal likert variables. British Journal of Mathematical and Statistical Psychology, 38, 171-189.

Paula, I. (2000). Habilidades sociales: educar hacia la autorregulación. Conceptualización, evaluación e intervención. Barcelona: Horsori.

Reyna, C. y Brussino, C. (2009). Propiedades Psicométricas de la Escala de Comportamiento Preescolar y Jardín Infantil en una Muestra de Niños Argentinos de 3 a 7 Años. Psykhe, 18(2), 127-140.

Reynolds, C.R. y Kamphaus, R.W. (1992). Behavior Assessment System for Children. Circle Pines: MN American Guidance Services.

Tomás, J. M. y Oliver, A. (2004). Análisis psicométrico confirmatorio de una medida multidimensional del autoconcepto en español. Revista Interamericana de Psicología, 38(2), 285-293.

Trianes, M.V., De la Morena, M.L. y Muñoz, A.M. (1999). Relaciones sociales y prevención de la inadaptación social y escolar. Archidona (Málaga): Ediciones Aljibe.

Trianes, M.V., Sánchez, A. y Muñoz, A. (2001). Educar la convivencia como prevención de la violencia interpersonal: perspectivas de los profesores. Revista Interuniversitaria de Formación del Profesorado, 41, 73-93.

Vallés, A. y Vallés, C. (1996). Las habilidades sociales en la escuela. Una propuesta curricular. Madrid: EOS. 
Análisis factorial confirmatorio de las subescalas del $P K B S-2$ para la evaluación de las habilidades sociales y los problemas de conducta en educación infantil

Walker, H.M. y McConnell, S.R. (1995). Walker-McConnell Scale of Social Competence and School Adjustment-Elementary Version. San Diego, C.A.: Singular Publishing Group.

Whiteside-Mansell, L., Bradley, R.H. y Mckelvey, L. (2009). Parenting and Preschool Child Development: Examination of Three Low-Income U.S. Cultural Groups. Journal of Child and Family Studies, 18, 48-60. 
Anexo: Preschool and Kindergaten Behavior Scale - Versión Española

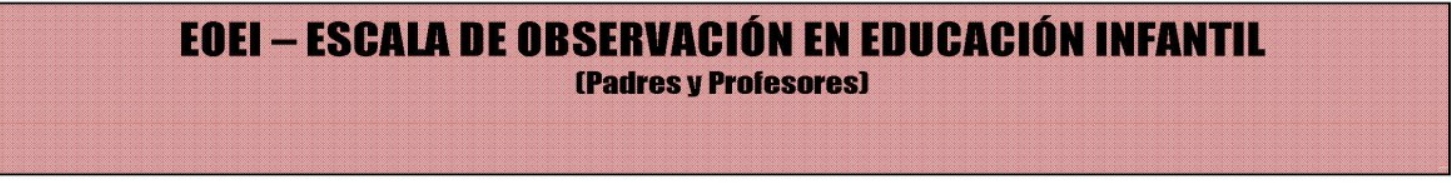

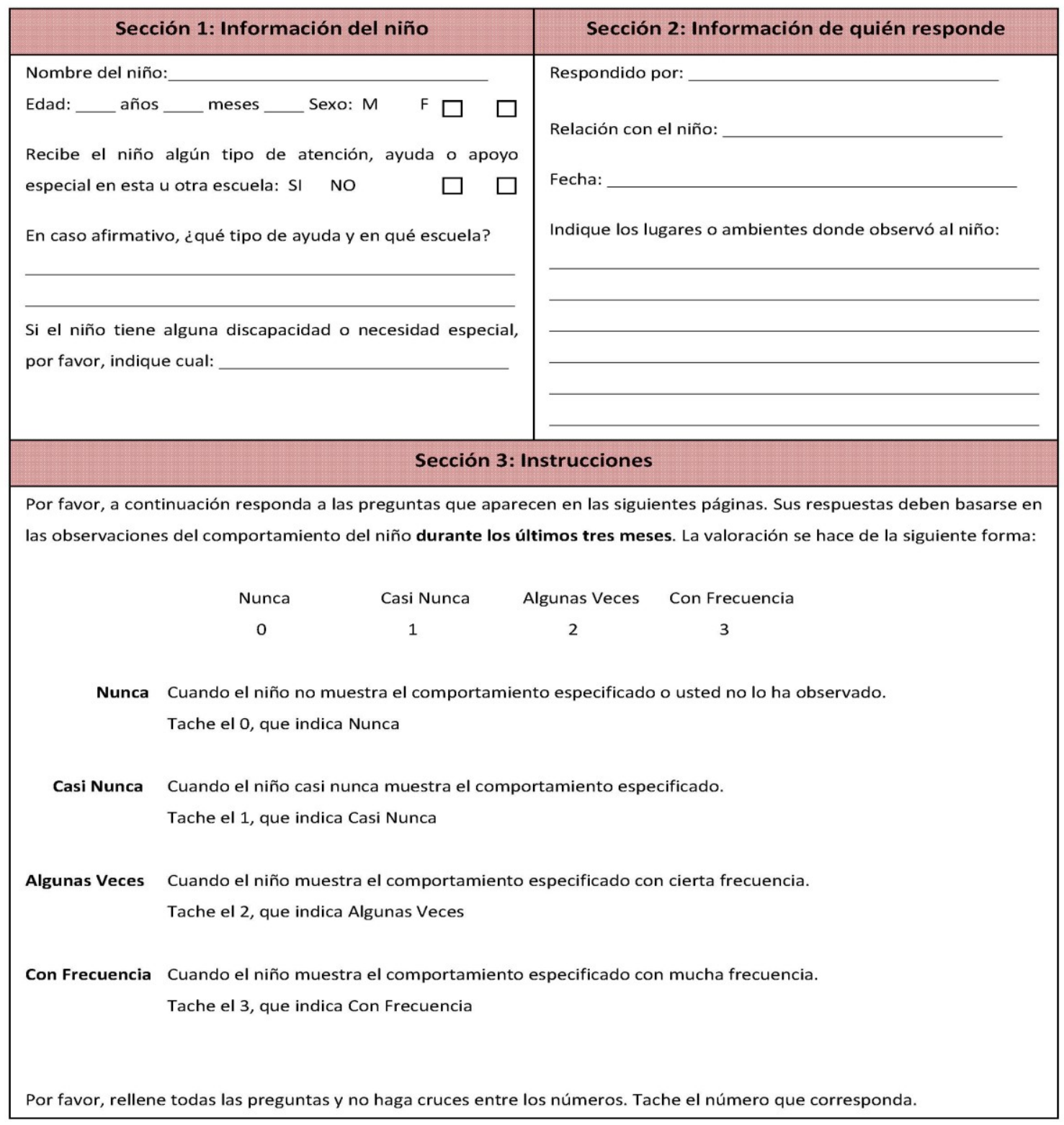


Análisis factorial confirmatorio de las subescalas del $P K B S-2$ para la evaluación de las habilidades sociales y los problemas de conducta en educación infantil

\section{Escala de Competencia Social}

\begin{tabular}{|c|c|c|c|c|c|c|c|c|}
\hline & & Nunca & Casi & Algunas & Con & \multicolumn{3}{|c|}{ Puntuación } \\
\hline 1. & Es independiente cuando juega y trabaja & 0 & 1 & 2 & 3 & & & \\
\hline 2. & Es cooperativo & 0 & 1 & 2 & 3 & & & \\
\hline 3. & Ríe y se divierte con otros niños & 0 & 1 & 2 & 3 & & & \\
\hline 4. & Juega con distintos niños & 0 & 1 & 2 & 3 & & & \\
\hline 5. & Intenta comprender lo que otros hacen (Ej.: ¿Por qué lloran?) & 0 & 1 & 2 & 3 & & & \\
\hline 6. & Es aceptado y cae bien a otros niños & 0 & 1 & 2 & 3 & & & \\
\hline 7. & Sigue las instrucciones de los adultos & 0 & 1 & 2 & 3 & & & \\
\hline 8. & Intenta hacer la tarea antes de pedir ayuda & 0 & 1 & 2 & 3 & & & \\
\hline 9. & Hace amigos fácilmente & 0 & 1 & 2 & 3 & & & \\
\hline 10. & Sabe controlarse & 0 & 1 & 2 & 3 & & & \\
\hline 11. & Otros niños le invitan a jugar & 0 & 1 & 2 & 3 & & & \\
\hline 12. & Utiliza bien su tiempo libre & 0 & 1 & 2 & 3 & & & \\
\hline 13. & Acepta separarse de los padres sin problemas & 0 & 1 & 2 & 3 & & & \\
\hline 14. & Participa en las conversaciones familiares o escolares & 0 & 1 & 2 & 3 & & & \\
\hline 15. & Pide ayuda a un adulto cuando la necesita & 0 & 1 & 2 & 3 & & & \\
\hline 16. & Cuando se leen historias se sienta y escucha & 0 & 1 & 2 & 3 & & & \\
\hline 17. & Defiende los derechos de otros niños & 0 & 1 & 2 & 3 & & & \\
\hline 18. & Se adapta bien a diferentes circunstancias & 0 & 1 & 2 & 3 & & & \\
\hline 19. & Tiene habilidades y capacidades que sus compañeros admiran & 0 & 1 & 2 & 3 & & & \\
\hline 20. & Anima a otros niños que se encuentran mal & 0 & 1 & 2 & 3 & & & \\
\hline 21. & Invita a otros niños a jugar & 0 & 1 & 2 & 3 & & & \\
\hline 22. & Recoge sus cosas cuando se le pide & 0 & 1 & 2 & 3 & & & \\
\hline 23. & Respeta las reglas & 0 & 1 & 2 & 3 & & & \\
\hline 24. & Busca el apoyo de un adulto cuando se encuentra mal & 0 & 1 & 2 & 3 & & & \\
\hline 25. & Comparte sus juguetes $y$ otras pertenencias & 0 & 1 & 2 & 3 & & & \\
\hline 26. & Defiende sus derechos & 0 & 1 & 2 & 3 & & & \\
\hline 27. & Se disculpa cuando molesta a otros sin querer & 0 & 1 & 2 & 3 & & & \\
\hline 28. & $\begin{array}{l}\text { Cuando es apropiado, cede y se compromete con las propuestas de } \\
\text { otros }\end{array}$ & 0 & 1 & 2 & 3 & & & \\
\hline 29. & Acepta las decisiones de los adultos & 0 & 1 & 2 & 3 & & & \\
\hline 30. & Acepta los turnos para utilizar los juguetes u otros objetos & 0 & 1 & 2 & 3 & & & \\
\hline 31. & Se muestra seguro en situaciones sociales & 0 & 1 & 2 & 3 & & & \\
\hline 32. & Reacciona correctamente cuando se le corrige & 0 & 1 & 2 & 3 & & & \\
\hline & Es sensible a los problemas de los adultos (Ej.: ¿percibe la tristeza?) & 0 & 1 & 2 & 3 & & & \\
\hline 34. & Muestra afecto por otros niños & 0 & 1 & 2 & 3 & & & \\
\hline & & & & & TOTALES & & & \\
\hline
\end{tabular}


Escala de Comportamiento Antisocial

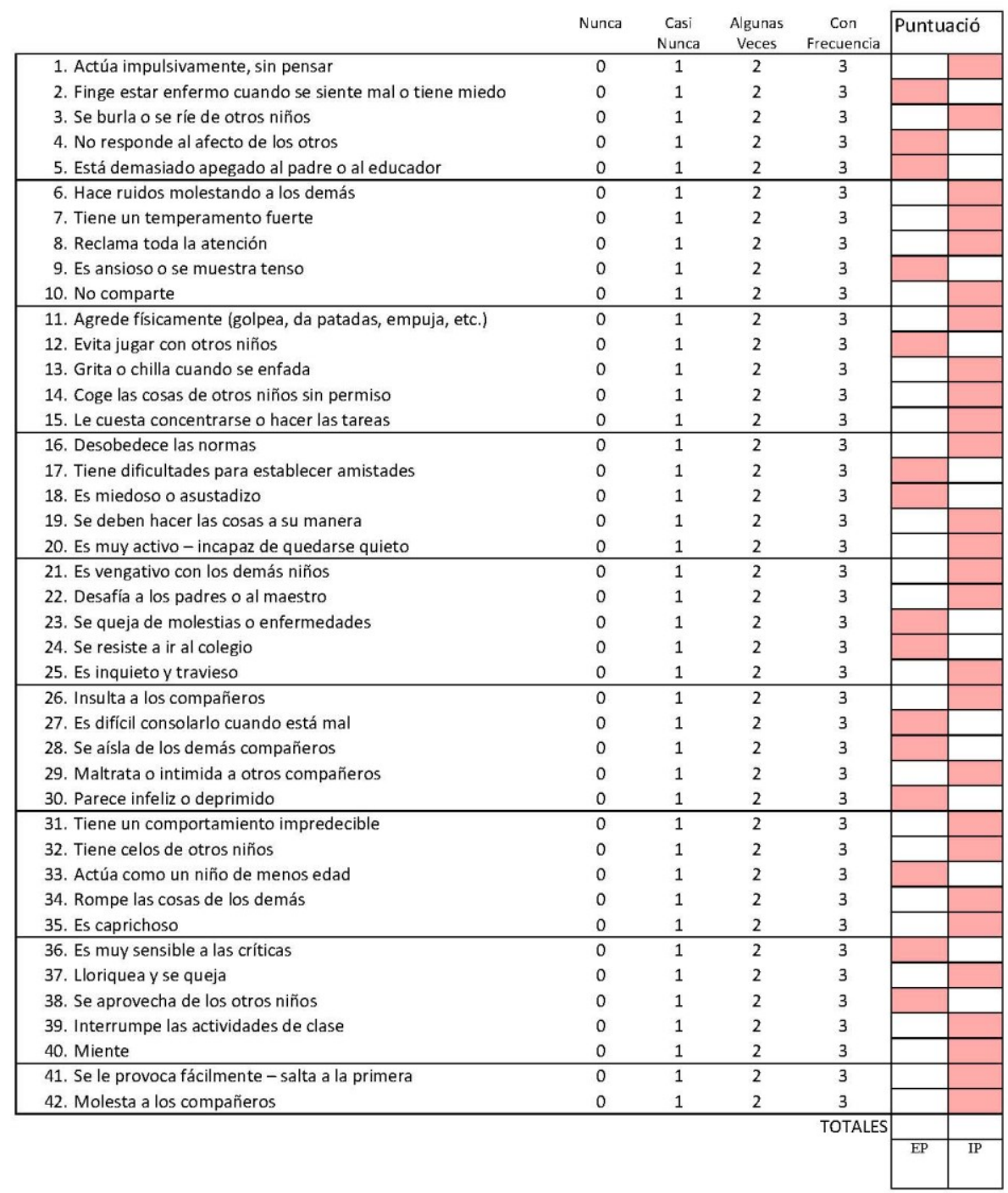

